Pacific Journal of Mathematics

VERSAL DETERMINANTAL DEFORMATIONS 


\title{
VERSAL DETERMINANTAL DEFORMATIONS
}

\author{
M. SCHAPS
}

The paper investigates the deformations of a determinantal scheme arising from a deformation of the defining matrix. A sufficient condition is given for the parameter space of the versal deformation space to contain a unique smooth subscheme parameterizing determinantal deformations. Examples are given in which various determinantal representations of a scheme give different determinantal deformation spaces.

Versal deformation spaces for schemes of codimension 1 or 2 are well-understood, but the theory for schemes of codimension 3 is much more difficult, and most of the calculated examples fall into a category which we will call pre-image schemes, $X=f^{-1}(Y)$ for $Y$ a rigid, CohenMacaulay scheme generated by minors of some generic matrix, with $V$ and $W$ ambiant affine spaces for $X$ and $Y$ and $\operatorname{codim}(X, V)=\operatorname{codim}(Y, W)$. The deformations of such schemes have been extensively studied for $Y$ which is the generic determinantal, symmetric determinantal, or Pfaffian scheme $[1,2,3,6]$. Of these the most important are the determinantal schemes and to simplify our discussion we will restrict our attention to that case, although the methods would apply as well to the other types of schemes.

Definition 1. If $X$ is a determinantal scheme of type $(a, b, c)$, with its ideal generated by the $c \times c$ minors of an $a \times b$ matrix $M$, then an $M$-deformation of $X$ is one obtained by deforming the entries of $M$. More generally, for a preimage scheme $f^{-1}(Y)$, an $f$-deformation of $X$ is one obtained by deforming the morphism $f$.

We will prove a sufficient condition for the versal $M$-deformation space to exist, and apply it to three of the important examples in which the versal deformation space is known: quotient singularities, monomial curves and coordinate axes.

EXAMPLE 1. The versal deformation space of the scheme of $n$ coordinate axes in $n$-space has been calculated by D. S. Rim. The ideal is generated by $x_{i} \cdot x_{j}, i \neq j$, where $x_{1}, \ldots, x_{n}$ are the coordinates. This is, in 
fact, a determinantal scheme, being given by the minors of any general matrix

$$
\left[\begin{array}{ll}
a_{1} x_{1} & a_{2} x_{2} \cdots a_{n} x_{n} \\
b_{1} x_{1} & b_{2} x_{2} \cdots b_{n} x_{n}
\end{array}\right] .
$$

Thus, as one would expect, the generic deformation is non singular. Rim has shown that we can take as parameters indeterminates $t_{i j}, \sum_{j \neq i} t_{i j}=0$, with deformed functions

$$
\left(x_{i}-t_{i j}\right)\left(x_{j}-t_{j i}\right)-\left(t_{i k}-t_{i j}\right)\left(t_{j k}-t_{j l}\right), \quad k \neq i, j,
$$

with relations determined by the requirement that the second factor be independent of the choice of $k$.

For $n=4$, codimension 3, these relations determine the cone of the Segre embedding of $P^{1} \times P^{3}$ into $P^{7}$, of dimension 5. Over the open dense set on which $t_{12}, t_{13}$ and $t_{14}$ are distinct, we in fact have the fibers all determinantal, as shown by the mapping

$$
k\left[t_{i j}\right] \rightarrow k[\alpha, \beta, \gamma] /(\alpha+\beta+\gamma)\left[\varepsilon_{1} \varepsilon_{2} \varepsilon_{3} \varepsilon_{4}\right]
$$

given by sending $t_{i j}$ to the appropriate entry in

$$
\left[\begin{array}{llll}
- & \alpha \varepsilon_{1} & \beta \varepsilon_{1} & \gamma \varepsilon_{1} \\
\alpha \varepsilon_{2} & - & \gamma \varepsilon_{2} & \beta \varepsilon_{2} \\
\beta \varepsilon_{3} & \gamma \varepsilon_{3} & - & \alpha \varepsilon_{3} \\
\gamma \varepsilon_{4} & \beta \varepsilon_{4} & \alpha \varepsilon_{3} & -
\end{array}\right]
$$

Computating shows that this is a homomorphism. When $\alpha, \beta, \gamma$ are all distinct, the deformed ideal in $k\left[t_{i j}, x\right]$ corresponds to the ideal generated by the minors of the matrix

$$
\left[\begin{array}{rl}
\varepsilon_{1} & x_{1} \\
x_{2}-\alpha \varepsilon_{2} & \alpha x_{2}+(\beta \gamma-\beta \alpha-\gamma \alpha) \varepsilon_{2} \\
x_{3}-\beta \varepsilon_{3} & \beta x_{3}+(\gamma \alpha-\gamma \beta-\alpha \beta) \varepsilon_{3} \\
x_{4}-\gamma \varepsilon_{4} & \gamma x_{4}+(\alpha \beta-\alpha \gamma-\beta \gamma) \varepsilon_{4}
\end{array}\right]
$$

Although the fibers are determinantal, the deformation itself is not determinantal, in that in addition to deforming the entries of the defining matrix of $X$ we must alter the defining matrix by varying $\alpha, \beta$ and $\gamma$ in order to obtain most of the deformations. Thus any theory for deforming determinantal schemes will have to take account of any nonuniqueness in the defining matrix which does not arise from ordinary row and column operations. 
The pathology exhibited here is non-generic, and we should expect a matrix sufficiently general to define a scheme with an essentially unique representation, and a versal deformation space.

Any matrix representation can be varied in two standard ways, without changing the resulting determinantal ideal. The simplest is to add to each entry of the matrix an element of the ideal. The second is to mimic an automorphism of the generic determinantal scheme, by multiplying on the left and right by invertible matrices. What is unusual in example 1 is the existence of alternative representations not obtained in this way. With this example as motivation, we make the following definition:

Definition 2. If $A^{\prime} \rightarrow A$ is a surjection of Artin rings with kernel $K$, $M_{1}, M_{2}$ matrices over $A^{\prime}[Z]$ with ideals $J_{1}, J_{2}$, such that $K J_{1}=K J_{2}$, and there exist $P \equiv I_{a}, Q \equiv I_{b}(\bmod K)$ such that $M_{2} \equiv P M_{1} Q\left(\bmod K J_{1}\right)$ then we say that $M_{1}$ and $M_{2}$ are equivalent over $A$.

If $K J_{1}=K J_{2}$ holds, then in fact $J_{1}=J_{2}$, since the differences between a given minor of $M_{1}$ and the corresponding minor in $M_{2}$ will be an element of $K J_{1}$.

Definition 3. If $M$ is a matrix over $A[Z]$, and if any two matrices lifting $M$ to $A^{\prime}[Z]$, which generate the same ideal, are equivalent, we say that $M$ has the unique lifting property for $A^{\prime} / A$. If this holds for every surjection $A^{\prime} \rightarrow A$, we simply say unique lifting property.

Note that if $A^{\prime \prime} \rightarrow A^{\prime} \rightarrow A$ is a composition of surjections, then in order to show that $M$ over $A[Z]$ has a unique lifting property for $A^{\prime \prime} / A$, it suffices to check it for $M$ for $A^{\prime} / A$ and for some lifting $M^{\prime}$ for $A^{\prime \prime} / A^{\prime}$. Then if $M_{1}, M_{2}$ over $A^{\prime \prime}[Z]$ reduce to $M_{1}^{\prime}, M_{2}^{\prime}$ over $A^{\prime}[Z]$ and $M$ over $A[Z]$, and the minors of $M_{1}$ and $M_{2}$ generate the same ideal $J^{\prime \prime}$, then we have

$$
P_{1}^{\prime} M_{1}^{\prime} Q_{1}^{\prime}+R_{1}^{\prime}=M^{\prime}=P_{2}^{\prime} M_{2}^{\prime} Q_{2}^{\prime}+R_{2}^{\prime}, \quad \text { with } R_{1}^{\prime}, R_{2}^{\prime} \equiv 0 \bmod K J^{\prime},
$$

and by taking liftings of $P_{l}^{\prime}, Q_{i}^{\prime}, R_{i}^{\prime}$, we get

$$
M_{1} \stackrel{A}{\sim} M_{1}^{\prime \prime} \stackrel{A^{\prime}}{\sim} M_{2}^{\prime \prime} \stackrel{A}{\sim} M_{2}
$$

Thus in order to check that $M$ has the unique lifting property, it is sufficient to check that each lifting of $M$ has the u.l.p. for small extensions, i.e. extensions whose kernel annihilates the maximal ideal.

Proposition 1. Suppose $X$ is a determinantal scheme with isolated singularity, and $M$ is a matrix representation with the unique lifting property. Then the functor of $M$-determinantal deformations of $X$ has a prorepresentable hull. 
Proof. We have to check the three conditions $H_{1}, H_{2}$ and $H_{3}$ from Schlessinger [7] for the subfunctor $F$ of $D_{B}$, with $F(A)$ being the set of isomorphism classes of flat deformations which are representable as determinantal deformations with respect to $M . F(k[\varepsilon]) \subset D_{B}(k[\varepsilon])$, so (H3) $\operatorname{dim} F(k[\varepsilon])<\infty$.

For (H1), we are given $A^{\prime \prime} \rightarrow A$, surjective, and $A^{\prime} \rightarrow A$. We need to show that

$$
F\left(A^{\prime} \underset{A}{X} A^{\prime \prime}\right) \rightarrow F\left(A^{\prime}\right) \underset{F(A)}{\times} F\left(A^{\prime \prime}\right)
$$

is surjective. Consider $\eta^{\prime} \in F\left(A^{\prime}\right), \eta^{\prime \prime} \in F\left(A^{\prime \prime}\right)$, both inducing $\eta \in F(A)$. Take determinantal representations $Y^{\prime}$ and $Y_{0}^{\prime \prime}$, with $Y=Y^{\prime} \times_{A^{\prime}} A, Y_{0}=$ $Y_{0}^{\prime \prime} \times_{A^{\prime \prime}} A$. We have $\theta: Y_{0} \stackrel{\sim}{\rightarrow} Y$, and since $A^{\prime \prime} \rightarrow A$ is surjective, this lifts to $\theta^{\prime \prime}: Y_{0}^{\prime \prime} \rightarrow Y^{\prime \prime}$. If $N^{\prime}, N_{1}^{\prime \prime}$ are the defining matrices of $Y^{\prime}, Y^{\prime \prime}$, they reduce to $N, N_{1}$ defining $Y$. Since both are liftings of $M$, which has the u.l.p., $N \stackrel{k}{\sim} N_{1}$. Thus

REMARK. When the hull exists, it provides a versal $M$-deformation, whose parameter space will in fact be smooth. This is an immediate consequence of the fact that all the generators and relations of a determinantal ideal are functions of the matrix entries. Hence, since both are deformed compatibly, every $M$-deformation is flat. The same holds for deformations of a fixed symmetric or Pfaffian representation of a preimage scheme.

Let $k[\varepsilon] \stackrel{\sim}{\rightarrow} k[T] / T^{2}$ be the ring of dual numbers. Suppose that $\varphi$ : $A^{\prime} \rightarrow k$ is a surjection of local Artin rings, with maximal ideal $m$ as kernel, and suppose that $\psi: A^{\prime} \rightarrow A$ is a small extension, so that a generator $t$ of the kernel satisfies $t m=0$.

There is a canonical isomorphism $\alpha: t A^{\prime}[Z] \stackrel{\sim}{\rightarrow} \varepsilon k[Z]=\varepsilon(k[\varepsilon][Z])$ induced by the composition of

$$
t A^{\prime} \stackrel{\sim}{\rightarrow} A^{\prime} / m=k \stackrel{\sim}{\rightarrow} \varepsilon k[\varepsilon] .
$$

Any matrix over $k[\varepsilon][Z]$ can be written uniquely as $M+\varepsilon N, M, N$ matrices over $k[Z]$.

Corollary. Let $M$ be an $a \times b$ matrix defined over $k[Z]$; let $J$ be the ideal of type $(a, b, c)$ generated by $J$, and suppose that $X=\operatorname{Spec}(k[Z] / J)$ has a versal deformation hull. Suppose that for every matrix $N$ over $k[Z]$ 
such that $M+\varepsilon N$ and $M$ generate the same ideal $J+\varepsilon J$ over $k[\varepsilon][Z]$, we can modify the rows and column of $N$ by suitable linear combinations of rows and columns of $M$, until all entries are in $J$. Then $X$ has a versal $M$-deformation hull.

Proof. By Proposition 1, it suffices to show that any such $M$ has the unique lifting property. As remarked immediately before the proposition, it suffices to investigate small extensions $\psi: A^{\prime} \rightarrow A$. Letting $M_{1}$ and $M_{2}$ be two matrices which generate the same determinantal ideal $J_{1}$, such that $M_{2}-M_{1} \equiv 0(\bmod (t))$, we apply the isomorphism $\alpha: t A^{\prime}[Z] \rightarrow \varepsilon k[Z]$ to obtain a unique matrix $N$ over $k[Z]$ such that if $N^{\prime}$ is any lifting to $A^{\prime}[Z]$,

$$
M_{2}=M_{1}+t N^{\prime} \text {. }
$$

For any subsets $\mu \subset\{1, \ldots, a\}$ and $\nu \subset\{1, \ldots, b\}$ of order $c$, let $M_{\mu \nu}$ be the submatrix of $M$ with these rows and columns. Then $\left|\left(M_{2}\right)_{\mu \nu}\right|-$ $\left|\left(M_{1}\right)_{\mu \nu}\right| \in t J_{1}$. For any $i \in \mu$, let $\hat{i}$ be the position of $i$ in $\mu$ considered as an ordered set, and similarly for any $j \in \nu$, let $\hat{j}$ be the position of $j$. Using the fact that $t^{2}=0$ and expanding the minors, with $b_{i j}^{\prime}=$ $(-1)^{i+j}\left|\left(M_{1}\right)_{\mu-\{i\} \nu-\{J\}}\right|$, and $b_{i j}$ corresponding maximal minors of $M$, we get

$$
\begin{aligned}
\left|\left(M_{2}\right)_{\mu \nu}\right|-\left|\left(M_{1}\right)_{\mu \nu}\right| & =t \sum_{i \in \mu} \sum_{j \in \nu} n_{i j}^{\prime} b_{i j}^{\prime} \stackrel{\alpha}{\rightarrow} \varepsilon \sum_{i \in \mu} \sum_{j \in \nu} n_{i j} b_{i j} \\
& =\left|(M+N)_{\mu \nu}\right|-\left|M_{\mu \nu}\right| .
\end{aligned}
$$

We conclude that the ideal generated by $M+\varepsilon N$ is contained in $J+\varepsilon J$, and since the differences between the generators all lie in $\varepsilon J$, a simple Nakayama type argument show that the ideals are, in fact, equal.

Applying the hypothesis of the proposition, we thus conclude that there exist matrices $R^{a \times a}$ and $S^{b \times b}$ such that

$$
M+\varepsilon N=(1+\varepsilon R) M(1+\varepsilon S) \quad(\bmod \varepsilon J)
$$

or

$$
\varepsilon N=\varepsilon(R M+M S) \quad(\bmod \varepsilon J) .
$$

Letting $P^{\prime}=1_{a}+t R^{\prime}$ and $Q^{\prime}=1_{b}+t S^{\prime}$ be the lifting induced by $\alpha^{-1}$ : $\varepsilon R \rightarrow t R^{\prime}, \varepsilon S \rightarrow t S^{\prime}$, we have

$$
t N^{\prime} \equiv t\left(R^{\prime} M_{1}+M_{1} S^{\prime}\right) \quad\left(\bmod t J_{1}\right) .
$$

Thus

$$
M_{2} \equiv P^{\prime} M_{1} Q^{\prime} \quad\left(\bmod t J_{1}\right)
$$

as required. 
Let $A_{\mu \nu}$ be the ideal generated by the $(c-1) \times(c-1)$ minors of $M_{\mu \nu}$. Let $B_{\mu \nu}$ be the ideal generated by those $c \times c$ minors $\left(M_{\mu^{\prime} \nu^{\prime}}\right)$ with

(a) $\mu=\mu^{\prime}$ and $\#\left(\nu-\nu^{\prime}\right) \leq 1$, or

(b) $\nu=\nu^{\prime}$ and $\#\left(\mu-\mu^{\prime}\right) \leq 1$,

and let $\bar{\Re}_{\mu \nu}$ be the ideal generated by the remaining $c \times c$ minors, so that $J=\Re_{\mu \nu}+\bar{\Re}_{\mu \nu}$. With this notation, we shall prove the following sufficient but not necessary condition:

Proposition 2. Let $X$ be a determinantal scheme of type $(a, b, c)$ with matrix $M$. If there exist sets $\mu$ and $\nu$ of $c$ elements, and subsets $\mu_{0} \subset \mu$ and $\nu_{0} \subset \nu$ of $c-1$ elements, such that

(1) $\mathbb{Q}_{\mu_{0} \nu_{0}} \neq\{0\}, \mathbb{Q}_{\mu \nu}$ is a determinantal ideal, that is, has height $(a-c+2)(b-c+2)$, and

(2) $\mathbb{Q}_{\mu^{\prime} \nu^{\prime}} \cap\left(\Re_{\mu^{\prime} \nu^{\prime}}+\overline{\mathscr{G}}_{\mu^{\prime} \nu^{\prime}}\right)=\Re_{\mu^{\prime} \nu^{\prime}}+\mathbb{Q}_{\mu^{\prime} \nu^{\prime}} \cdot \overline{\mathscr{G}}_{\mu^{\prime} \nu^{\prime}}$ for $\mu^{\prime}=\mu, \mu_{0}$ and $\nu^{\prime}=$ $\nu, \nu_{0}$ then $X$ has a versal determinantal deformation.

Proof. Let $b_{i j}$ be the minor $(-1)^{i+\jmath}\left|\left(M_{\mu \nu}\right)_{i j}\right|$. Then the $\mu \nu$ minor of $M+\varepsilon N$ lies in $J$ only if

$$
b=\sum_{i j} n_{i j} b_{i j} \in J .
$$

Thus $b$, which represents the change in the $\mu \nu$ minor induced by the deformation of $M$ by $N$, lies in $\mathbb{Q}_{\mu \nu} \cap\left(\mathscr{B}_{\mu \nu}+\overline{\mathscr{B}}_{\mu \nu}\right)$. Therefore, by hypothesis (2), there are elements $a_{\bar{\mu} \bar{\mu}}$ and $n_{i j}{ }^{\prime}$ such that $b=\sum a_{\bar{\mu} \bar{\nu}} \cdot\left|M_{\bar{\mu} \bar{\nu}}\right|$ $+\sum n_{i j^{\prime}}, b_{\imath \jmath}$, with $n_{i j^{\prime}} \in \bar{\Re}_{\mu \nu}$, with $(\bar{\mu}, \bar{\nu})=\left(\mu, \nu-\{j\} \cup\left\{j^{\prime}\right\}\right)$ or $(\bar{\mu}, \bar{\nu})=$ $\left(\mu-\{i\} \cup\left\{i^{\prime}\right\}, N\right)$ of order $c$. By adding $a_{\bar{\mu} \bar{\nu}}$ times the $j^{\prime}$ column of $M$ to the $j$ th column of $N$, or $a_{\vec{\mu} \bar{\nu}}$ times the $i^{\prime}$ row of $M$ to the $i$ th row of $N$ respectively, the first sum can be eliminated. The second sum is eliminated by adding $n_{i j^{\prime}} \in J$ to $n_{i j}$. Thus we may presume $b=0$. Then $\left[n_{i j}\right]$ is a relation on the minors $\left[b_{i j}\right]$ generating the determinantal ideal $\mathbb{Q}_{\mu \nu}$ of type $(c, c, c-1)$, so $n_{\imath \jmath}$ is generated by row and column operations, the generating relations being the scalar product of a row or column with the minors of a $c \times c-1$ or $(c-1) \times c$ submatrix containing it, or the difference between two expansions of the determinant. Thus we may reduce to the case $n_{i j}=0$ for $i \in \mu$ and $j \in \nu$.

Let $\nu=\nu_{0} \cup\left\{i^{\prime \prime}\right\}$, and $\mu=\mu_{0} \cup\left\{j^{\prime \prime}\right\}$, so that, for example $\mathscr{Q}_{\mu \nu_{0}}$ is generated by the minors $b_{l J^{\prime \prime}}$ of $M_{\mu \nu}$.

We now expand $\left|M_{\mu \nu^{\prime}}+\varepsilon N_{\mu \nu^{\prime}}\right|$, where $\nu^{\prime}=\nu_{0} \cup\left\{j^{\prime}\right\}$, for $j^{\prime} \notin \nu$. This lies in $J$ if and only if $s_{j^{\prime}}$ does, where

$$
s_{J^{\prime}}=\sum_{i \in \mu} n_{l j^{\prime}} b_{i j^{\prime \prime}} \in \mathbb{Q}_{\mu \nu_{0}} .
$$


Thus since $\mathscr{Q}_{\mu \nu_{0}} \cap J=\mathscr{B}_{\mu \nu_{0}}+\mathscr{Q}_{\mu \nu_{0}} \cdot \overline{\mathscr{Q}}_{\mu \nu_{0}}$, we have

$$
s_{J^{\prime}}=\sum_{\bar{\nu}=\nu_{0} \cup\{\bar{j}\}} a_{\mu \bar{\nu}}\left|M_{\mu \bar{\nu}}\right|+\sum_{i=1}^{c} b_{i j^{\prime \prime}} n_{i j^{\prime}}^{\prime},
$$

where $n_{i j^{\prime}} \in \overline{\mathscr{B}}_{\mu \nu_{0}}$. Thus if for each $\bar{\nu}$ we subtract from $j^{\prime}$ the column $\bar{j}$ times $\mathbb{Q}_{\mu \bar{\nu}}$, and subtract $n_{l j^{\prime}}^{\prime}$, from each $n_{l j^{\prime}}$, we have a new $N$ for which $s_{j^{\prime}}$ vanishes. The equation $(*)$ above then determines a relation on the $b_{\imath j^{\prime \prime}}$, so the $j^{\prime}$ column of $N$ must be a combination of the columns in $\nu_{0}$, and thus can be eliminated by column operations. We may thus assume $n_{i j}=0$ for $i \in \mu$. By performing the corresponding operations on the rows outside of $\mu$, we may also assume that $n_{l j}=0$ for $j \in \nu$.

Letting $\mu^{\prime}=\mu_{0} \cup\left\{i^{\prime}\right\}$ and $\nu^{\prime}=\nu_{0} \cup\left\{j^{\prime}\right\}$, the assumption that $\mid M_{\mu^{\prime} \nu^{\prime}}$ $+\varepsilon N_{\mu^{\prime} \nu^{\prime}} \mid \in J$ leads to an equation $s_{l^{\prime} j^{\prime}}=n_{l^{\prime} j^{\prime}} b_{t^{\prime \prime} j^{\prime \prime}} \in J$. Since $\mathscr{G}_{\mu^{\prime} v^{\prime}}$ is the zero ideal, and $b_{l^{\prime \prime} J^{\prime \prime}} \neq 0$ is not a zero divisor, we must have $n_{l^{\prime} \jmath^{\prime}} \in \overline{\mathscr{S}}_{\mu^{\prime} \nu^{\prime}}$, and thus can be eliminated. We thus reduce $N$ to the zero matrix, as desired.

REMARK. For the generic determinantal scheme the conclusion of the lemma is that the infinitesimal automorphisms of $Y$ are determined by the $W$-points of $\mathcal{G}=\mathrm{GL}(a, k) \times \mathrm{GL}(b, k)$. For the generic $2 \times n$ matrix, to

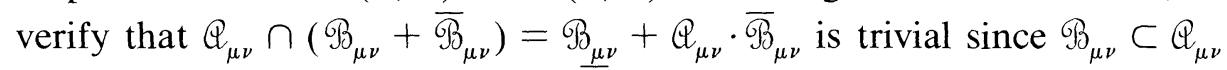
as always, and in this case $\mathbb{Q}_{\mu \nu}$ and $\mathscr{\mathscr { S }}_{\mu \nu}$ have no common variables, so that

$$
\mathbb{Q}_{\mu \nu} \cap \overline{\mathscr{B}}_{\mu \nu}=\mathbb{Q}_{\mu \nu} \cdot \overline{\mathscr{B}}_{\mu \nu} \text {. }
$$

The proposition shows that a sufficiently generic determinantal scheme has a versal deformation space of determinantal deformations. This can be presumably shown for Pfaffians with Pfaffian deformations and for symmetric determinantal schemes and their symmetric deformations as well.

To show that the condition in the lemma is not necessary, simply consider any curve in three space, which is determinantal of type ( $n$, $n-1, n-1)$. The first condition is not fulfilled because one cannot have $\mathbb{Q}_{\mu \nu}$ of height 4 .

EXAMPLE 2. Let $X$ be the surface with quotient singularity (see $[3,4]$ ) given by dividing $C^{2}$ by the action $u \rightarrow i u, v \rightarrow i v . X$ can be embedded in $\mathbf{C}^{5}$, and has a representation as a determinantal scheme given by the $2 \times 2$ minors of the matrix,

$$
\left[\begin{array}{llll}
x_{0} & x_{1} & x_{2} & x_{3} \\
x_{1} & x_{2} & x_{3} & x_{4}
\end{array}\right]=M_{1}
$$


the codimension being $(2-1)(4-1)=3$ as required. Let $Y_{1} \subset W_{1}$ be the generic determinantal scheme generated by the $2 \times 2$ minors of a $2 \times 4$ matrix, and let

$$
f_{1}: k\left[w_{1}, \ldots, w_{8}\right] \rightarrow k\left[x_{0}, \ldots, x_{4}\right]
$$

be given by mapping each $w_{1}$ to the corresponding matrix entry. Then $X=f_{1}^{-1}(Y)$ we can take $\nu=\{1,3\}$, giving the regular sequence $\left\{x_{0}, x_{1}, x_{2}, x_{3}\right\}$ and $\mu=\{1,2\}, \mu_{0}=\nu_{0}=1$.

However, $X$ has a second non-equivalent representation. Let $Y_{2} \subset W_{2}$ be the scheme determined by the vanishing of the $2 \times 2$ minors of the generic $3 \times 3$ symmetric matrix.

$Y_{2}$ is Cohen-Macaulay of codimension 3, and the following mapping exhibits $X$ as a $\left(Z, W_{2}\right)$ preimage scheme with representation $\left(f_{2}, Y_{2}\right)$,

$$
\left[\begin{array}{lll}
w_{11} & w_{12} & w_{13} \\
w_{12} & w_{22} & w_{23} \\
w_{13} & w_{23} & w_{33}
\end{array}\right] \stackrel{f_{2}}{\rightarrow}\left[\begin{array}{lll}
x_{0} & x_{1} & x_{2} \\
x_{1} & x_{2} & x_{3} \\
x_{2} & x_{3} & x_{4}
\end{array}\right]=M_{2} .
$$

In fact, $X$ is isomorphic to a section of $Y_{2}$ by the hyperplane $w_{22}=w_{13}$.

How are these two very different representations reflected in the tangent space of the versal deformation space? It has two components. One of dimension 3 corresponds to the versal $M_{1}$-deformation.

$$
\left[\begin{array}{llll}
x_{0} & x_{1} & x_{2} & x_{3} \\
x_{1}+t_{1} & x_{2}+t_{2} & x_{3}+t_{3} & x_{4}
\end{array}\right] .
$$

The other, of dimension 1, corresponds to the versal $M_{2}$-deformation

$$
\left[\begin{array}{lll}
x_{0} & x_{1} & x_{2}-s \\
x_{1} & x_{2} & x_{3} \\
x_{2}-s & x_{3} & x_{4}
\end{array}\right]
$$

The situation in this example is rare but illuminating. It is rare that a scheme is representable as a preimage of two different rigid schemes, or even in two essentially different ways as the preimage of the same scheme. It is illuminating in leading us to expect that first order deformations which are hybrids of deformations by distinct representations will be obstructed.

In the quotient singularities of codimension 3, the v.d.s. has a parameter space with two components, one of dimension 1 which is determiantal and simultaneously resolvable and one of dimension 3. In the special case treated by Pinkham in his thesis these correspond to a determinantal and a symmetric determinantal versal deformation. The symmetric determinantal representation occurs for the action with $n=8, q=3$, i.e. 
$u \rightarrow \zeta u, v \rightarrow \zeta^{3} v$, and $\zeta^{8}=1$, where the invariant monomials can be arranged in a symmetric $3 \times 3$ matrix

$$
\left[\begin{array}{lll}
u^{8} & u^{5} v & \left(u^{2} v^{2}\right)^{2} \\
u^{5} v & u^{2} v^{2} & u v^{5} \\
\left(u^{2} v^{2}\right)^{2} & u v^{5} & v^{8}
\end{array}\right]
$$

There does not seem to be a determinantal representation in this case.

EXAmple 3. Consider the curve defined by the vanishing of the $2 \times 2$ minors of the matrix

$$
\left[\begin{array}{lll}
x_{1} & x_{2} & x_{3} \cdots x_{g+1} \\
x_{2} & x_{3} & x_{4} \cdots x_{1}^{2}
\end{array}\right]
$$

discussed in the section on monomial curves in Pinkham [3]. It has a versal determinantal deformation space, since we can take $\mu_{0}=\{1\} \subset \mu$ $=\{1,2\}$ and $\nu_{0}=\{1\} \subset \nu=\{1,3\}$ and the hypotheses of the lemma will be satisfied. However unlike the case in Example 2 the corresponding smooth subscheme of the parameter space is not a component.

\section{REFERENCES}

1. H. Kleppe, Deformations of schemes defined by vanishing of Pfaffians, University of Oslo, (1977).

2. D. Laksov, Deformations of determinantal schemes, Comp. Math., 30 (1975), 273-292.

3. H. Pinkham, Deformations of algebraic varieties with $G_{m}$ action, Asterique, no. 20, Soc. Math. Franc., (1974).

4. O. Riemenschneider, Deformationen von Quotienten-singularitaten (nach zyklischen Gruppen), Math. Ann., 209 (1974), 211-248.

5. M. Schaps, Non-singular deformations of space curves, using determinantal schemes, dissertation, Harvard University, (1972).

6. __ Non-singular deformations of a determinantal scheme, Pacific J. Math., 65, No. 1, (1976).

7. M. Schlessinger, Functors of Artin rings, Trans. Amer. Math. Soc., 130 (1968), 208-222.

Received April 20, 1981.

BAR-ILAN UNIVERSITY

RAMAT GAN, ISRAEL 



\section{PACIFIC JOURNAL OF MATHEMATICS}

\section{EDITORS}

Donald BabbitT (Managing Editor)

University of California

Los Angeles, CA 90024

Hugo Rossi

University of Utah

Salt Lake City, UT 84112

C. C. Moore and Arthur Ogus

University of California

Berkeley, CA 94720
J. DugunduI

Department of Mathematics

University of Southern California

Los Angeles, CA 90089-1113

R. FinN and H. SAmelson

Stanford University

Stanford, CA 94305

\section{ASSOCIATE EDITORS}
R. ARENS
E. F. BECKENBACH
B. H. NEUMANN
F. WOLF
K. YosHIDA (1906-1982)

\section{SUPPORTING INSTITUTIONS}

UNIVERSITY OF ARIZONA

UNIVERSITY OF BRITISH COLUMBIA

CALIFORNIA INSTITUTE OF TECHNOLOGY

UNIVERSITY OF CALIFORNIA

MONTANA STATE UNIVERSITY

UNIVERSITY OF NEVADA, RENO

NEW MEXICO STATE UNIVERSITY

OREGON STATE UNIVERSITY
UNIVERSITY OF OREGON

UNIVERSITY OF SOUTHERN CALIFORNIA

STANFORD UNIVERSITY

UNIVERSITY OF HAWAII

UNIVERSITY OF TOKYO

UNIVERSITY OF UTAH

WASHINGTON STATE UNIVERSITY

UNIVERSITY OF WASHINGTON 


\section{Pacific Journal of Mathematics}

Vol. 107, No. $1 \quad$ January, 1983

John Kelly Beem and Phillip E. Parker, Klein-Gordon solvability and the

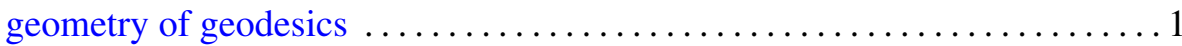

David Borwein and Amnon Jakimovski, Transformations of certain

sequences of random variables by generalized Hausdorff matrices ..... 15

Willy Brandal and Erol Barbut, Localizations of torsion theories . . . . . . . 227

John David Brillhart, Paul Erdős and Richard Patrick Morton, On sums

of Rudin-Shapiro coefficients. II ........................... 39

Martin Lloyd Brown, A note on tamely ramified extensions of rings $\ldots \ldots \ldots 71$

Chang P'ao Ch'ên, A generalization of the Gleason-Kahane-Żelazko

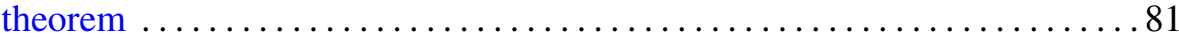

I. P. de Guzman, Annihilator alternative algebras $\ldots \ldots \ldots \ldots \ldots \ldots$. . . . 89

Ralph Jay De Laubenfels, Extensions of $d / d x$ that generate uniformly

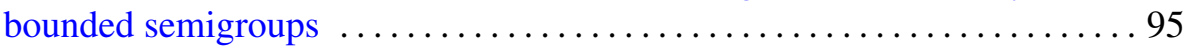

Patrick Ronald Halpin, Some Poincaré series related to identities of $2 \times 2$

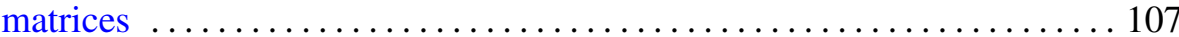

Fumio Hiai, Masanori Ohya and Makoto Tsukada, Sufficiency and

relative entropy in $*$-algebras with applications in quantum systems . . . 117

Dean Robert Hickerson, Splittings of finite groups $\ldots \ldots \ldots \ldots \ldots \ldots \ldots 14$

Jon Lee Johnson, Integral closure and generalized transforms in graded

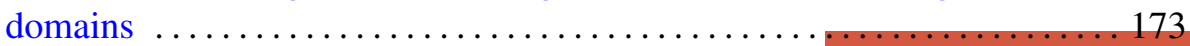

Maria Grazia Marinari, Francesco Odetti and Mario Raimondo, Affine

curves over an algebraically nonclosed field . ................. 179

Douglas Shelby Meadows, Explicit PL self-knottings and the structure of

PL homotopy complex projective spaces $\ldots \ldots \ldots \ldots \ldots \ldots \ldots \ldots \ldots$

Charles Kimbrough Megibben, III, Crawley's problem on the unique

$\omega$-elongation of $p$-groups is undecidable .................... 205

Mary Elizabeth Schaps, Versal determinantal deformations $\ldots \ldots \ldots \ldots 213$

Stephen Scheinberg, Gauthier's localization theorem on meromorphic

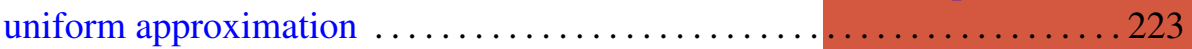

Peter Frederick Stiller, On the uniformization of certain curves . . . . . . . 229

Ernest Lester Stitzinger, Engel's theorem for a class of algebras . . . . . . . . 245

Emery Thomas, On the zeta function for function fields over $F_{p} \ldots \ldots \ldots 251$ 